\title{
Myofibroblasts and their resistance to apoptosis: a possible mechanism of osteoradionecrosis
}

This article was published in the following Dove Press journal:

Clinical, Cosmetic and Investigational Dentistry

12 July 2012

Number of times this article has been viewed

Lei Tian'

Li-Sheng $\mathrm{He}^{\prime}$

Bhavesh Soni ${ }^{2}$

Hong-Tao Shang'

'Department of Oral and Maxillofacial Surgery, School of Stomatology, Fourth Military Medical University, China; ${ }^{2}$ Maxillofacial Unit, Morriston Hospital, Swansea, Wales, UK
Correspondence: Hong-Tao Shang Department of Oral and Maxillofacial Surgery, School of Stomatology, Fourth Military Medical University, 145 West Changle Road, Xi'an, 7I0032, China

Tel +86298477 6109

Fax +86298477353।

Email tianlei007@yahoo.com

\begin{abstract}
Osteoradionecrosis (ORN) in the head and neck area is the most devastating long-term complication of radiotherapy, with slow progression and inability to heal spontaneously. ORN can lead to intolerable pain, fractures, and sequestration of devitalized bone and fistulae, making oral feeding impossible and causing facial deformation. In spite of its notorious reputation over at least 90 years, the precise pathogenesis of ORN has not been fully clarified, which has led to obstacles in the management of the disease. Several theories about its pathogenesis have been formulated, and radiation-induced fibrosis is the newest one. According to this theory, ORN is essentially a type of fibrosis induced by radiotherapy, and antifibrosis therapy has been shown to be effective in its treatment. We assumed that ORN, like fibrosis in other organs, is the result of a process of fibrogenesis in which myofibroblasts are the key effector cells. The uninterrupted accumulation of myofibroblasts and consequent persistent excess production of collagenous extracellular matrix and tensile force result in loss of normal function and ultimately radiation-induced fibrosis. During this process, myofibroblasts may be protected from apoptosis by acquiring an immune-privileged capacity, which allows continuous matrix synthesis. If this hypothesis proves to be correct, it would enable better understanding of the cellular and molecular mechanisms underlying the pathogenesis and progression of ORN, and would help improve our ability to prevent occurrence of ORN, give an earlier diagnosis, and treat it more effectively.
\end{abstract}

Keywords: osteoradionecrosis, apoptosis, fibrosis, mandible, myofibroblast

\section{Introduction}

Head and neck cancers have a global annual incidence of about 560,000 new cases, and cause approximately 300,000 deaths per year throughout the world. ${ }^{1}$ While radical surgery is the most important treatment among the comprehensive therapies for head and neck cancers, radiotherapy has been extensively used as primary therapy, adjuvant to surgery, in conjunction with concurrent chemotherapy, or as palliative treatment for late-stage and unresectable malignancies. ${ }^{2}$ Although radiotherapy has been shown to lead to an approximately $10 \%$ absolute increase in 5-year cancer-specific and overall survival, ${ }^{3}$ the patient is susceptible to side effects, including tooth decay, mucositis, dysphagia, xerostomia, dermatitis, and, worst of all, osteoradionecrosis (ORN) of the jaw. ${ }^{4}$

ORN, first described by Regaud in $1922,{ }^{5}$ is a chronic disease process with devitalization and devascularization of bone due to irradiation, and does not tend to heal spontaneously. ${ }^{6}$ Obliterative endarteritis, hyperemia, hyalinization, cellular loss, hypovascularization, thrombosis, and fibrosis are common histologic findings in ORN. 
This can lead to intolerable pain, pathologic fracture, trismus, ulceration, sequestration of devitalized bone, and fistulae, making oral feeding impossible and sometimes even leading to death. ${ }^{6,7}$ Despite advances in radiotherapy technique and increased attention to predisposing factors in recent years, there is no reliable evidence showing that the incidence of ORN has decreased, much less been totally eliminated. ${ }^{7}$ An investigation in a large study population of 830 patients over a period of 30 years showed that the overall incidence of ORN was $8.2 \%,{ }^{8}$ and a recent investigation showed that the incidence in the mandible is as high as $40 \%$ at 5 years. ${ }^{4}$ Because of the relatively high associated morbidity and severe clinical symptoms, doctors have been trying to find effective methods to prevent and cure ORN for decades. Unfortunately, treatment remains unsatisfactory, and even the etiopathogenesis and definition of ORN are still not agreed on by consensus.

Deeper understanding of the pathogenesis of ORN would be most helpful in developing strategies for prophylaxis of ORN and improve its prognosis. Since ORN was described, several theories have been proposed and each has led to sequential changes in treatment. The radiation, trauma, and infection theory was proposed by Meyer and Dannenberg in $1970 .{ }^{9}$ This theory suggested that injury provided the opportunity for invasion of oral microbiologic flora into the underlying irradiated bone, which led to ORN being referred to as secondary infection after injury to devitalized bone, or as radiation-induced osteomyelitis. This theory lasted for a decade and became the foundation of antibiotic therapy for ORN. However, this theory was later brought into question, in view of the failure to demonstrate bacterial invasion in compromised bone and an unsatisfactory curative effect. Marx proposed the hypoxic-hypocellular-hypovascular theory as a new understanding of the pathophysiology of osteoradionecrosis. ${ }^{10}$ He concluded that ORN was a complex metabolic and homeostatic deficiency in tissues created by radiationinduced cellular injury. Radiation-induced hypoxia and ischemia could result in an imbalance between cell death and cell replacement, as well as between collagen degradation and synthesis, leading to a chronic nonhealing wound. This theory formed the cornerstone for the use of hyperbaric oxygen in the treatment of ORN and still dominates. Although many clinical guidelines mention the possibility of using hyperbaric oxygen therapy as a coadjuvant measure for treating ORN in minor cases or in the early stages, its clinical value remains the subject of considerable debate. ${ }^{11,12}$ Until now, no consensus has existed regarding either the mechanism of action or the effectiveness of hyperbaric oxygen in the prevention and treatment of ORN. A randomized, placebo-controlled, double-blind study of hyperbaric oxygen for treating overt ORN of the mandible showed no benefits of hyperbaric oxygen over placebo in recovery, and neither did it slow the progression of disease or relieve pain. ${ }^{13}$ Recent papers have shown that radiogenic cellular effects on bone occur earlier than the well known vascular alterations, and there is comparable transcutaneous $\mathrm{pO}_{2}$ in irradiated and nonirradiated skin, which also challenged Marx's theory. ${ }^{14}$

In recent years, a new theory for the pathogenesis of ORN has arisen, suggesting that ORN is a radiation-induced fibroatrophic disease. ${ }^{15,16}$ Cells in the radiated region are damaged as a result of acute inflammation, with free radical formation, microvascular thrombosis, chronic activation of fibroblasts by a series of growth factors, and finally, bone and tissue necrosis. According to this theory, the key event in the progression of ORN is activation and dysregulation of fibroblastic activity, which leads to atrophic tissues within a previously irradiated area. Apart from the nature and timing of stimuli, the pathophysiology process of ORN is almost similar to fibrosis occurring in the lung, liver, vessels, or kidney, which can be induced by a number of factors, including injury, drugs, virus infections, autoimmune reactions, and alcohol. Some antifibrosis medicines, eg, pentoxifylline and tocopherol (vitamin E), have been used to treat ORN, with dramatic results. ${ }^{17,18}$

Given that radiation-induced fibroatrophy or radiationinduced fibrosis is essentially a type of fibrosis, it should share analogous pathophysiologic processes with other fibrosis diseases. It is now widely agreed that the myofibroblast is the cell type most responsible for accumulation of the interstitial matrix and the consequent structural deformations associated with fibrosis. ${ }^{19}$ Myofibroblasts have been identified in few normal tissues, and their function in normal tissues has been little explored. On the other hand, it has been confirmed that the myofibroblast plays a pivotal role in pathologic tissues, including hypertrophic scarring, fibromatosis, fibrocontractive diseases, and the stromal reaction to epithelial tumors. ${ }^{20}$

Myofibroblasts can be derived from heterogeneous origins and activated by biological and mechanical stimuli. Once activated, the myofibroblast acquires a phenotype characterized by excessive production of collagenous extracellular matrix and tensile force, which endows on itself a critical role in resolution of inflammation and scar formation during wound healing to maintain tissue integrity. ${ }^{19,20}$ In normal conditions, when stimulating factors have gone, the myofibroblast will disappear during extensive apoptosis at the end of tissue repair 
and remodeling. However, in the development of hypertrophic scars and other fibrotic diseases, myofibroblasts acquire a capacity for uninterrupted accumulation and persistent excess production of extracellular matrix and tensile force. ${ }^{21-23}$ This phenomenon has been investigated extensively, and lack of apoptosis by myofibroblasts has now been suggested as the most likely mechanism for fibrogenesis in the liver, lung, and kidney, as well as during atheromatous plaque formation, which eventually result in complete loss of organ function. ${ }^{19,24}$ Although some researchers have been investigating the function of myofibroblasts in radiation fibrosis, the evidence for the myofibroblast being key in the pathogenesis of radiation fibrosis are few and obscure. Further, no literature has been published about the relationship between myofibroblasts and ORN as yet. Could myofibroblasts be the main effector cells in ORN, like in other fibrosis diseases? This paper focuses on this issue and attempts to establish a novel hypothesis regarding the role of the myofibroblast in the development of ORN and tries to clarify the possible pathophysiologic mechanism.

\section{Hypothesis}

We propose the hypothesis that the myofibroblast is the key effector cell in the pathogenesis of ORN, as with fibrosis in other organs. ORN should not be regarded as radiation fibrosis being only involved with bone, but also with all other tissues in the radiated area, including skin, vessels, and muscles. Myofibroblasts may derive from resident fibroblasts, epithelial cells, endothelial cells, smooth muscle cells, and bone marrow cells in the irradiated region. Myofibroblasts can also become resistant to apoptosis, which enables their uninterrupted accumulation and long-term existence. With high rates of proliferation, excess secretion of extracellular matrix protein, and increased tensile force, the balance between synthesis and degradation is destroyed in irradiated tissues, resulting in a reduction in the bony matrix and its replacement with fibrous tissues. Ultimately, even decades after radiotherapy, these irradiated areas remain fragile, and are still subject to late physicochemical stimuli, so have a tendency to develop ORN.

\section{Possible differentiation of myofibroblasts in ORN}

The myofibroblast, which has a spindle or stellate shape, as well as contractile, secretory, and macrophage properties, is not a typical component of normal tissues, and can only be found in granulation tissue, non-neoplastic fibrosing conditions, and tumor stroma. ${ }^{25}$ The myofibroblast is essentially a reactive cell, which means it can be differentiated by its heterogeneous origins, depending on the physiologic or pathologic situation. These precursors in fibrosis include local fibroblasts, epithelial cells, endothelial cells, smooth muscle cells, pericytes, hepatic perisinusoidal cells or stellate cells, as well as bone marrow-derived cells. ${ }^{19,25}$ Local fibroblasts are believed to represent the major source of myofibroblasts in most situations. ${ }^{20}$ The role of epithelial mesenchymal transition and its specific form of endothelial-mesenchymal transition have been demonstrated to exist and to contribute to progression of cardiac, lung, liver, and corneal fibrosis. ${ }^{26,27}$ Pericytes and smooth muscle cells from the vasculature have also been found to serve as myofibroblast progenitors and have been suggested to contribute to fibrosis in scleroderma, atherosclerosis, and radiation-induced fibrosis of the gastrointestinal tract. ${ }^{21,28}$ In addition, bone marrow-derived cells known as fibrocytes have been suggested to represent an alternative source of myofibroblasts during skin wound healing and in liver, lung, and kidney fibrosis, as well as in the stromal reaction to epithelial tumors. ${ }^{29}$ Except for some specific cell types, such as hepatic stellate cells or alveolar septal cells, most of the potential progenitor cells mentioned above exist in the maxillofacial region, which provides cytologic evidence for differentiation of myofibroblasts in ORN.

The differentiation of myofibroblasts is a key event in the pathogenesis of fibrosis, but the underlying mechanism is complex. It has been demonstrated that inflammation accompanies most fibrotic states and plays an initiating role. Under the influence of numerous growth factors, cytokines, hormones released by inflammatory and other cell types, precursor cells are activated and differentiate into myofibroblasts. ${ }^{30}$ It is likely that the heterogeneity of myofibroblast origin requires specific factors and specific mechanical conditions in each situation, but regardless, transforming growth factor-beta 1 (TGF- $\left.\beta_{1}\right)$, which is produced by a wide range of inflammatory, endothelial, and epithelial cell types, is the principal growth factor responsible for differentiation of myofibroblasts. TGF- $\beta_{1}$ activates progenitors by binding and activating specific plasma membrane receptors, and by activating its downstream target signal, connective tissue growth factor, to promote differentiation and proliferation of myofibroblasts. ${ }^{20}$ In addition to TGF- $\beta_{1}$, other cytokines such as interleukin-13, interleukin-4, basic fibroblast growth factor, and platelet-derived growth factor, also help in this process and sustain fibrogenesis. ${ }^{31,32}$

Apart from inflammation and inflammatory cytokines, there are other factors inducing myofibroblast differentiation in fibrosis. Microvascular injury is regarded as the 
prominent profibrogenesis factor in systemic sclerosis and diabetic nephropathy. ${ }^{28,33}$ In these diseases, damage to vascular endothelium by autoantibodies stimulates the release of chemokines and other factors, including endothelin-1, platelet-derived growth factor, basic fibroblast growth factor, and vascular endothelial growth factor, which cause the vessel wall to become more permeable and promote recruitment and proliferation of inflammatory cells and differentiation into myofibroblasts. Hypoxia secondary to microvascular damage is also regarded as a possible mechanism of fibrosis in human interstitial renal tubular fibrosis. ${ }^{34}$

Excess reactive oxygen species (ROS) and oxidative stress are postulated to play roles in the initiation and perpetuation of a wide range of fibrotic diseases, including atherosclerosis, cardiac fibrosis, idiopathic lung fibrosis, and systemic sclerosis. ROS can upregulate the expression of several fibrogenic genes by activating hypoxia-inducible factor 1 alpha and release-activated TGF- $\beta_{1}$ from extracellular reservoirs by ROS-mediated oxidation of a methionine residue in the latency-associated protein. ${ }^{35}$ In fibrosis associated with a prominent adaptive immune response, ROS is believed to direct the differentiation of CD4+ T cells towards the Thy-2 lymphocyte phenotype, which is characterized by release of strongly profibrotic cytokines, ie, interleukin-4 and interleukin-13. ${ }^{36}$ High levels of ROS oxidize the phagocytosed lipid and stimulate fibrogenic cytokines inducing some progenitor cells, eg, smooth muscle cells in atherosclerosis, to undergo differentiation or transition to myofibroblasts.

The pathogenesis and differentiation of myofibroblasts in ORN are still not clarified. However, research on radiationinduced fibrosis in the skin, lung, and rectum has revealed some pathophysiologic characteristics. After radiation, immediate oxidative damage to DNA, proteins, and lipids are the initiating chemical events which lead to cell injury or death, and the acute inflammatory responses in the endothelium are proposed as important determinants of radiation fibrosis. ${ }^{37}$ Chemokines, which are released in response to radiation injury, attract leukocytes to the site of injury, thereby triggering an inflammatory response, and generate further release of multiple cytokines, including tumor necrosis factor alpha, platelet-derived growth factor, basic fibroblast growth factor, interleukin-1, interleukin-4, interleukin-6, TGF- $\beta_{1}$, and, more recently identified, connective tissue growth factor. According to the immunocytochemical report by Canney and Dean, ${ }^{38}$ TGF- $\beta_{1}$ is considered to be the main cytokine involved in radiation-induced fibrosis. Another confirmed important factor in the development of radiation-induced fibrosis is ROS, with its function of profibrosis. Injured endothelial cells generate further release of ROS from polymorphs and other phagocytes. ROS can release activated TGF- $\beta_{1}$ from extracellular reservoirs and other cytokines, resulting in unregulated fibroblastic activation and persistence of the myofibroblast phenotype. ${ }^{35}$

It is well known that radiation can lead to vascular injury, which is a prominent feature of radiation injury and a possible primary pathogenic signal that precedes fibrosis. The vascular injury includes epithelial cell injury and loss of this as a natural barrier, thrombosis and microvascular atrophy, and luminal stenosis of the large vessels, may lead to necrosis of the microvessels, local ischemia, and secondary hypoxia. It has been verified that changes in the permeability of the vessel wall and hypoxia could promote release of a series of cytokines and activation of myofibroblasts, possibly by activated hypoxia-inducible factor 1 alpha and TGF- $\beta_{1}$ pathways. ${ }^{37,39}$

Compared with normal fibrosis, the pathophysiologic processes of ORN, although not quite clear yet, are very similar. Pathologic events, including the inflammatory reaction, release of multiple cytokines, in particular TGF- $\beta_{1}$, injury to the vasculature and secondary hypoxia, and ROS or oxidative stress, commonly exist and are believed to function as profibrogenesis factors. These events likely provide the pathophysiologic evidence for differentiation of myofibroblasts in ORN.

\section{Capacity for resistance to apoptosis}

The myofibroblast is essential for the integrity of the mammalian body by virtue of its role in wound-healing. ${ }^{20}$ Under normal conditions, myofibroblasts play an essential role in the resolution of inflammation and scar formation. After recovery from injury, myofibroblasts subsequently disappear by apoptosis from the injured site to maintain tissue homeostasis. When the apoptotic mechanism fails, prolonged formation and accumulation of extracellular matrix lead to such conditions as hypertrophic scar and keloid or fibrosis in different organs. ${ }^{25}$ Resistance of myofibroblasts to apoptosis has been demonstrated in systemic sclerosis, idiopathic pulmonary fibrosis, and liver fibrosis, and shown to be the main mechanism of fibrogenesis. In radiation-induced fibrosis, myofibroblasts have also been found to be persistent during the constitutive fibrotic phase and to correspond with the histopathologic description of hypercellularized fibrosis areas and with the clinical observation of radiation-induced fibrous swelling. ${ }^{39}$ There has been no clear evidence indicating that myofibroblasts in ORN can resist apoptosis until now, but in view of the theory of radiation-induced fibrosis, we have 
reason to believe that the fate of myofibroblasts in ORN has much in common with other types of fibrosis.

How myofibroblasts obtain this capability is not fully clear, and some hypotheses have been proposed when trying to explore the underlying mechanism. An ability of myofibroblasts to evade immune surveillance is one of them, and has been investigated extensively. Some recent experiments have indicated that myofibroblasts possess the ability to not only evade death but also actively promote apoptosis of T lymphocytes via the Fas/FasL pathway, which allows them to escape immune surveillance, resulting in organ fibrosis such as sclerosis or lung fibrosis. ${ }^{40}$ Could these findings be transferred to ORN? Certainly, the possible mechanism needs to be studied further.

Since 2004, pentoxifylline and tocopherol have been shown to be effective in treating radiation tissue injury and established ORN. ${ }^{17}$ Pentoxifylline can exert an antitumor necrosis factor alpha effect, increase erythrocyte flexibility, and inhibit inflammatory reactions and production of extracellular matrix. Vitamin E can scavenge reactive oxygen species, as well as inhibit TGF- $\beta_{1}$ and expression of procollagen genes. These two drugs act synergistically as potent antifibrotic agents. Interestingly, pentoxifylline and tocopherol have both been shown to be able to induce apoptosis of malignant cells. Pentoxifylline can trigger a series of events leading to apoptosis in cutaneous T cell lymphoma cells by upregulating expression of Fas and TRAIL, and by enhancing FasL-mediated killing in T cell lymphoma. ${ }^{41}$ Specific forms of tocopherol display potent apoptotic activity against a wide range of cancer cell types, while having little or no effect on normal cell function or viability. ${ }^{42}$ It is also well known that evasion of immune surveillance is a classic mechanism of tumor formation in cancer cells, which have been shown to resist Fas-induced apoptosis. Possibly, this is also evidence that myofibroblasts in ORN can resist apoptosis and perpetuate themselves by evasion of immune surveillance, like cancer cells.

\section{Future research}

Our hypothesis indicates that myofibroblasts and their resistance to apoptosis might be the main pathophysiologic mechanism in ORN. Before we can confirm this, there are still some questions to resolve. Firstly, we have to show that myofibroblasts do exist in established ORN. These myofibroblasts could be isolated from zones of fibrosis in patients with ORN and be cultivated by primary cell culture. Further, given that there is a risk of poor wound healing if surgery is performed in patients with ORN, established animal models of ORN could be used for isolation, cultivation, and identification of myofibroblasts. ${ }^{43}$

Morphologic observation and immunohistochemical examination of distinctive proteins, such as $\alpha$-smooth muscle actin, vimentin, and EDA cellular fibronectin, are the main techniques for identification of myofibroblasts. Secondly, apoptosis of myofibroblasts should be shown to be decreased in ORN. Myofibroblasts isolated and cultured from patients with ORN or animal models of ORN could be used to determine if decreased apoptosis occurs. If this is the case, further studies should be undertaken to clarify the underlying mechanism.

The proapoptotic pathway for myofibroblasts should also be investigated in animal models of ORN to determine if there is a possibility for prevention or reversal of ORN, which could further strengthen our hypothesis and identify a potential treatment for ORN. Some strategies have been developed to increase apoptosis of myofibroblasts experimentally and been shown to have an effect of antifibrogenesis in liver and lung fibrosis and in scleroderma. ${ }^{44-46}$ These strategies include specific delivery of apoptosis-inducing drugs (eg, single-chain antibody to an extracellular domain of a myofibroblast membrane protein), using protein kinase inhibitors (phosphatidylinositol 3-kinase or focal adhesion kinase) as specific inducers of apoptosis in myofibroblasts, and some cytokines (eg, hepatocyte growth factor).

\section{Consequences of a novel hypothesis}

ORN is a devastating complication of radiotherapy after treatment of head and neck cancer. While predisposing factors are clearly evident, the pathophysiologic process of osteoradionecrosis is still not clarified completely, and there is ongoing debate regarding the best treatment. Based on recent advances in cellular and molecular biology, a new theory of radiation-induced fibroatrophy has been developed. We have focused on the myofibroblast, which exists in all types of fibrosis and is widely assumed to be the key effector cell involved in development of ORN. Activation of the myofibroblast and its defective apoptosis might be the overriding factor in the pathogenesis of ORN.

Ours is a novel explanation for the pathogenesis of ORN, and if confirmed, might create an opportunity to establish a noninvasive prediction system with the cytologic characteristics of myofibroblasts, and could even create a reliable method to evaluate the risk of ORN, monitor its development, and evaluate the effects of therapeutic agents.

This novel hypothesis could also create an opportunity to develop a therapeutic protocol for ORN. Some strategies have 
been proposed for the prevention and treatment of fibrosis, but depend on a deeper understanding of the pathophysiological mechanism of fibrosis. However, there are a multitude of pathways ranging from the initial pathologic stimulus to abnormal collagen synthesis, offering a bewildering range of therapeutic targets, and possibly with uncertain therapeutic effects. Antibodies against TGF- $\beta_{1}$ have long been shown to reduce bleomycin-induced lung fibrosis in mice, ${ }^{47}$ but a randomized clinical trial of recombinant human TGF- $\beta_{1}$ antibody (CAT-192) in patients has failed to demonstrate any therapeutic effect on human fibrosis so far, and has had adverse effects. ${ }^{48}$ This reflects the pleiotropic effects of TGF- $\beta_{1}$ on a wide range of cell types, and the complex interactions between different cytokines, which have made TGF- $\beta_{1}$-targeted therapy difficult. In addition, ORN is usually diagnosed when tissue destruction is well underway, and it is likely that future therapies would need to target an established resident myofibroblast population. Therefore, inducing disappearance of myofibroblasts by stimulating them to enter the apoptosis pathway may be a promising therapeutic strategy in ORN. Moreover, proapoptotic therapy in ORN might have the potential benefit of preventing tumor recurrence, but this needs further investigation.

In summary, the present hypothesis reflects a novel view of the pathophysiologic mechanism of ORN, and further research on this hypothesis will help us to gain an insight into the pathogenesis of ORN, and could pave the way for more effective therapy in the future.

\section{Disclosure}

The authors report no conflicts of interest in this work.

\section{References}

1. St Guily JL, Borget I, Vainchtock A, Rémy V, Takizawa C. Head and neck cancers in France: an analysis of the hospital medical information system (PMSI) database. Head Neck Oncol. 2010;2:22-30.

2. Ko C, Citrin D. Radiotherapy for the management of locally advanced squamous cell carcinoma of the head and neck. Oral Dis. 2009;15: 121-132.

3. Chen P, Shieh T, Ho P, et al. Prognostic factors associated with the survival of oral and pharyngeal carcinoma in Taiwan. BMC Cancer. 2007;7:101-113.

4. Monnier Y, Broome M, Betz M, Bouferrache K, Ozsahin M, Jaques B. Mandibular osteoradionecrosis in squamous cell carcinoma of the oral cavity and oropharynx: incidence and risk factors. Otolaryngol Head Neck Surg. 2011;144:726-732.

5. Regaud C. Sur la necrose des os attenté par un processus cancereux et traites par les radiaions [Osteonecrosis during the process of cancer's treatment by radiations]. Compt Rend Soc Biol. 1922;87:629. French.

6. Jereczek-Fossa BA, Orecchia R. Radiotherapy-induced mandibular bone complications. Cancer Treat Rev. 2002;28:65-74.

7. Lee IJ, Koom WS, Lee CG, et al. Risk factors and dose-effect relationship for mandibular osteoradionecrosis in oral and oropharyngeal cancer patients. Int J Radiat Oncol Biol Phys. 2009;75:1084-1091.
8. Reuther T, Schuster T, Mende U, Kübler A. Osteoradionecrosis of the jaws as a side effect of radiotherapy of head and neck tumour patients a report of a thirty year retrospective review. Int J Oral Maxillofac Surg. 2003;32:289-295.

9. Meyer OT, Dannenberg AM Jr. Radiation, infection, and macrophage function. II. Effect of whole body radiation on the number of pulmonary alveolar macrophages and their levels of hydrolytic enzymes. J Reticuloendothel Soc. 1970;7:79-90.

10. Marx RE. A new concept in the treatment of osteoradionecrosis. J Oral Maxillofac Surg. 1983;41:351-357.

11. Spiegelberg L, Djasim UM, van Neck HW, Wolvius EB, van der Wal KG. Hyperbaric oxygen therapy in the management of radiation-induced injury in the head and neck region: a review of the literature. $J$ Oral Maxillofac Surg. 2010;68:1732-1739.

12. Shaw RJ, Dhanda J. Hyperbaric oxygen in the management of late radiation injury to the head and neck. Part I: Treatment. Br J Oral Maxillofac Surg. 2011;49:2-8.

13. Annane D, Depondt J, Aubert P, et al. Hyperbaric oxygen therapy for radionecrosis of the jaw: a randomized, placebo-controlled, double-blind trial from the ORN96 study group. J Clin Oncol. 2004; 22:4893-4900.

14. Rudolph R, Tripuraneni P, Koziol JA, McKean-Matthews M, Frutos A. Normal transcutaneous oxygen pressure in skin after radiation therapy for cancer. Cancer. 1994;74:3063-3070.

15. Madrid C, Abarca M, Bouferrache K. Osteoradionecrosis: an update. Oral Oncol. 2010;46:471-474.

16. Chrcanovic BR, Reher P, Sousa AA, Harris M. Osteoradionecrosis of the jaws - a current overview - Part 1: physiopathology and risk and predisposing factors. Oral Maxillofac Surg. 2010;14:3-16.

17. Delanian S, Lefaix JL. Complete healing of severe osteoradionecrosis with treatment combining pentoxifylline, tocopherol and clodronate. Br J Radiol. 2002;75:467-469.

18. Chrcanovic BR, Reher P, Sousa AA, Harris M. Osteoradionecrosis of the jaws - a current overview - Part 2: dental management and therapeutic options for treatment. Oral Maxillofac Surg. 2010;14:81-95.

19. Kis K, Liu X, Hagood JS. Myofibroblast differentiation and survival in fibrotic disease. Expert Rev Mol Med. 2011;13:e27.

20. Hinz B. Formation and function of the myofibroblast during tissue repair. J Invest Dermatol. 2007;127:526-537.

21. Coen M, Gabbiani G, Bochaton-Piallat ML. Myofibroblast-mediated adventitial remodeling: an underestimated player in arterial pathology. Arterioscler Thromb Vasc Biol. 2011;31:2391-2396.

22. Sarrazy V, Billet F, Micallef L, Coulomb B, Desmoulière A. Mechanisms of pathological scarring: role of myofibroblasts and current developments. Wound Repair Regen. 2011;19 Suppl 1:s10-s15.

23. Parola M, Pinzani M. Hepatic wound repair. Fibrogenesis Tissue Repair. 2009;2:4-11.

24. Aarabi S, Bhatt KA, Shi Y, et al. Mechanical load initiates hypertrophic scar formation through decreased cellular apoptosis. FASEB J. 2007;21:3250-3261.

25. Hinz B, Phan SH, Thannickal VJ, Galli A, Bochaton-Piallat ML, Gabbiani G. The myofibroblast: one function, multiple origins. $\mathrm{Am} J$ Pathol. 2007;170:1807-1816.

26. Zeisberg M, Kalluri R. The role of epithelial-to-mesenchymal transition in renal fibrosis. J Mol Med. 2004;82:175-181.

27. Piera-Velazquez S, Li Z, Jimenez SA. Role of endothelial-mesenchymal transition (EndoMT) in the pathogenesis of fibrotic disorders. Am J Pathol. 2011;179:1074-1080.

28. Schrimpf C, Duffield JS. Mechanisms of fibrosis: the role of the pericyte. Curr Opin Nephrol Hypertens. 2011;20:297-305.

29. Forbes SJ, Russo FP, Rey V, et al. A significant proportion of myofibroblasts are of bone marrow origin in human liver fibrosis. Gastroenterology. 2004;126:955-963.

30. Eyden B. El miofibroblasto en tejidos normales y en situaciones patológicas [The myofibroblast in health and disease]. Rev Esp Patol. 2008;41:3-10. Spanish. 
31. Saito A, Okazaki H, Sugawara I, Yamamoto K, Takizawa H. Potential action of IL-4 and IL-13 as fibrogenic factors on lung fibroblasts in vitro. Int Arch Allergy Immunol. 2003;132:168-176.

32. Perl AK, Gale E. FGF signaling is required for myofibroblast differentiation during alveolar regeneration. Am J Physiol Lung Cell Mol Physiol. 2009;297:L299-L308.

33. Kelly KJ, Dominguez JH. Treatment of the post-ischaemic inflammatory syndrome of diabetic nephropathy. Nephrol Dial Transplant. 2010;25:3204-3212.

34. Toullec A, Gerald D, Despouy G, et al. Oxidative stress promotes myofibroblast differentiation and tumour spreading. EMBO Mol Med. 2010;2:211-230.

35. На H, Lee HB. Reactive oxygen species amplify glucose signalling in renal cells cultured under high glucose and in diabetic kidney. Nephrology (Carlton). 2005;10:S7-S10.

36. Tang JT, Fang JY, Gu WQ, Li EL. T cell immune response is correlated with fibrosis and inflammatory activity in hepatitis B cirrhotics. World J Gastroenterol. 2006;12:3015-3019.

37. Yarnold J, Brotons MC. Pathogenetic mechanisms in radiation fibrosis. Radiother Oncol. 2010;97:149-161.

38. Canney PA, Dean S. Transforming growth factor beta: a promotor of late connective tissue injury following radiotherapy? $\mathrm{Br} J$ Radiol. 1990;63:620-623.

39. Gervaz P, Morel P, Vozenin-Brotons MC. Molecular aspects of intestinal radiation-induced fibrosis. Curr Mol Med. 2009;9:273-280.

40. Wallach-Dayan SB, Golan-Gerstl R, Breuer R. Evasion of myofibroblasts from immune surveillance: a mechanism for tissue fibrosis. Proc Natl Acad Sci U S A. 2007;104:20460-20465.
41. Rishi L, Gahlot S, Kathania M, Majumdar S. Pentoxifylline induces apoptosis in vitro in cutaneous T cell lymphoma (HuT-78) and enhances FasL mediated killing by upregulating Fas expression. Biochem Pharmacol. 2009; 77:30-45.

42. Wang $\mathrm{P}, \mathrm{Yu} \mathrm{W}, \mathrm{Hu} Z$, et al. Involvement of $\mathrm{JNK} / \mathrm{p} 73 / \mathrm{NOXA}$ in vitamin $\mathrm{E}$ analog-induced apoptosis of human breast cancer cells. Mol Carcinog. 2008;47:436-445.

43. Cohen M, Nishimura I, Tamplen M, et al. Animal model of radiogenic bone damage to study mandibular osteoradionecrosis. Am JOtolaryngol. 2011;32:291-300.

44. Sosa Seda IM, Mott JL, Akazawa Y, et al. NOXA mediates hepatic stellate cell apoptosis by proteasome inhibition. Hepatol Res. 2010;40: 701-710.

45. Garneau-Tsodikova S, Thannickal VJ. Protein kinase inhibitors in the treatment of pulmonary fibrosis. Curr Med Chem. 2008;15: 2632-2640.

46. Garcia-Gonzalez E, Selvi E, Balistreri E, et al. Cannabinoids inhibit fibrogenesis in diffuse systemic sclerosis fibroblasts. Rheumatology (Oxford). 2009;48:1050-1056.

47. Giri SN, Hyde DM, Hollinger MA. Effect of antibody to transforming growth factor beta on bleomycin induced accumulation of lung collagen in mice. Thorax. 1993;48:959-966.

48. Denton CP, Merkel PA, Furst DE, et al. Recombinant human antitransforming growth factor betal antibody therapy in systemic sclerosis a multicenter, randomized, placebo-controlled phase I/II trial of CAT-192. Arthritis Rheum. 2007;56:323-333.
Clinical, Cosmetic and Investigational Dentistry

\section{Publish your work in this journal}

Clinical, Cosmetic and Investigational Dentistry is an international, peer-reviewed, open access, online journal focusing on the latest clinical and experimental research in dentistry with specific emphasis on cosmetic interventions. Innovative developments in dental materials, techniques and devices that improve outcomes and patient satisfac-

\section{Dovepress}

tion and preference will be highlighted. The manuscript management system is completely online and includes a very quick and fair peerreview system, which is all easy to use. Visit http://www.dovepress. com/testimonials.php to read real quotes from published authors. 